\title{
Sonographic approach to the tumours of retroperitoneal space
}

\author{
${ }^{\star}$ Victor Schiopu, Vasile Turcanu, Nicolai Ghidirim
}

\author{
Department of Oncology \\ Nicolae Testemitanu State University of Medicine and Pharmacy, Chisinau, the Republic of Moldova
}

Authors' ORCID iDs, academic degrees and contributions are available at the end of the article

*Corresponding author - Victor Schiopu, e-mail: victor.schiopu@usmf.md

Manuscript received November 01, 2021; revised manuscript December 10, 2021; published online December 17, 2021

\begin{abstract}
Background: Timely diagnosis of primary retroperitoneal tumours is one of the current challenges of clinical oncology. This is due to the rarity, polymorphism and diagnostic difficulties of primitive retroperitoneal tumours.

Material and methods: The study is cross-sectional, prospective and retrospective. The study group is represented by 118 patients with abdominal and retroperitoneal space tumours. Using the receiver operating characteristic (ROC) analysis curve and calculating the average quality of the diagnostic model, the informativeness of ultrasonography in the diagnosis of primary retroperitoneal tumours (PRT) was appreciated.

Results: For tumour localization, the ultrasonography (USG) as a diagnostic model demonstrated an appropriate use criteria (AUC) of 0.641 (95\% CI $0.541,0.740, \mathrm{p}<0.001$ ), and the mean quality of the diagnostic model was 0.54 . Following the statistical analysis, was found a partial correlation between the size of the tumour and the dimensions estimated at USG of 0.540 ( $95 \%$ CI $0.295,0.737, \mathrm{p}<0.001$ ), which represents a high positive correlation. To determine the uni- or multicentric character of the tumour, the USG demonstrated an integrative value of sensitivity and specificity of 0.644 (95\% CI $0.415,0.873, p<0.001$. In assessing the proximity ratio of retroperitoneal tumours, the highest AUC was recorded in the assessment of the ratio of tumour to pancreas - 0.838 (95\% CI 0.705.0.971, p <0.001) and kidney - $0.861(95 \%$ CI $0.699,1.024, \mathrm{p}<0.001)$.

Conclusions: Ultrasonography is a fairly informative imaging diagnostic method in the diagnosis of retroperitoneal tumours. The characteristics of the tumours obtained after the ultrasound examination provide indirect information about the malignant or benign nature of the primitive tumour, which allows the assessment of the next stages of diagnosis and treatment.

Key words: ultrasonography, retroperitoneal tumours.
\end{abstract}

\section{Cite this article}

Schiopu V, Turcanu V, Ghidirim N. Sonographic approach to the tumours of the retroperitoneal space. Mold Med J. 2021;64(6):37-40. https://doi. org/10.52418/moldovan-med-j.64-6.21.07.

\section{Introduction}

Primary retroperitoneal tumours (PRTs) are an extremely heterogeneous group of tumours of mesenchymal, neuroectodermal or vestigial origin and occur in the retroperitoneum. PRTs can be benign or malignant, the most common being retroperitoneal sarcoma. Approximately $70-80 \%$ of primary retroperitoneal soft tissue tumours are malignant; however, they represent only $0.1-0.2 \%$ of all malignancies $[1,2]$. Retroperitoneal sarcomas (RPS) are rare tumours of mesenchymal origin. The incidence of these tumours is difficult to determine, being 0.31 per 100000 people per year. About $53-56 \%$ of patients are women, and the average age at diagnosis is 59-61 years old [3,4].

Timely diagnosis of PRTs is a challenge for clinicians due to the rarity of this pathology and the difficulty of diagnosing these tumours. The difficulties of diagnosis are due to the peculiarities of the late clinical manifestation of retroperitoneal tumours, which are exaggeratedly large at the time of diagnosis.

The use of ultrasonography with Doppler technique significantly improves the early differential diagnosis of non- organ tumours of the peritoneal cavity and retroperitoneal space.

Data on the semiology of PRTs are not sufficiently systematized. This is due to the low incidence and lack of mainstream clinical trials to systematize the data on this topic. Definition of the diagnostic criteria for the malignant or benign type of tumours requires further research.

The aim of the study was to evaluate the informativeness of ultrasonography as a method of imaging diagnosis in primitive retroperitoneal tumours.

\section{Material and methods}

This is a complex study, prospective and retrospective structural analysis of clinical, imaging, morpho-pathological and immunohistochemical data of 118 patients with tumours of peritoneal cavity and retroperitoneal space investigated and treated at the Institute of Oncology of the Republic of Moldova, 2015-2020.

To determine the informativeness of the investigation method used, the representative study group was calculated in the EpiInfo 7.2.2.6 Program, "StatCalc-Sample Size and 
Power" section for cross-sectional study based on the following parameters:

- 95.0\% confidence interval for significance of results,

- Statistical power - 80.0\%,

- Frequency $0.01-0.2 \%$ is on average up to $1.0 \%$.

Ultrasonography of the abdominal cavity and retroperitoneal space was performed in $2 \mathrm{D}$, colour and spectral Doppler to assess the status of the tumour against the main blood vessels (abdominal aorta, renal arteries, inferior vena cava, portal system, etc.) or to determine tumour vascularization. If the tumour was in the small pelvis, the sonographic examination was performed with a full bladder.

The final diagnosis was established by morphological or immunohistochemical examination of the removed tumour or bioptate taken by diagnostic laparotomy or by ultrasound guided biopsy. The statistical processing of the data obtained in the study was performed following the unanimously accepted principles.

Descriptive statistics were used for both categorical and nominal parameters, represented by absolute and relative frequencies, supplemented by $95 \%$ confidence intervals, and for continuous, mean, median, standard deviation and percentile parameters (25\% and $75 \%$ ).

The evaluation of ultrasonography as the diagnostic method used in the study, as well as of the imaging semiology, was performed by the receiver operating characteristics (ROC) analyses curve and by determining the average quality of the diagnostic model.

In addition, some statistical tests were performed for the independent groups, the procedures being selected according to the level of measurement of the studied parameters, some particularities of the studied data and the distribution of continuous data. Thus, the $\chi 2$ test with continuity corrections, the Fisher test and the Mann Whitney test were used.

The obtained data was processed using IBM / PC, using the statistical processing software "Statistical Package for the Social Sciences" SPSS 17 for Windows 10.0.5 (SPSS, Chicago, IL, USA) and GraphPad PRISM ${ }^{\circledR} 5.0$ for Windows 5.0 (GraphPad Software, Inc.).

\section{Results}

Although the initial study group consisted of 118 patients, the status of primary retroperitoneal tumour was confirmed morphopathologically and immunohistochemically in only $84(71.18 \%)$ of them, $34(28.81 \%)$ of the patients had retroperitoneal metastases or organic tumours. Imaging evaluation of all patients included in the study was performed by ultrasonography of peritoneal cavity and retroperitoneal space, and pelvic cavity using the contrast CT.

Among the patients with a confirmed diagnosis of primary retroperitoneal tumour, $36(42.9 \%)$ were men and 54 $(57.1 \%)$ were women, the mean age was 57 years $(\sigma=12.0)$, $\mathrm{Me}=59$.

Although, in most cases, the PRTs are malignant, they metastasize quite rarely, but they have a high recurrence rate. Thus, among the patients with PRTs included in the

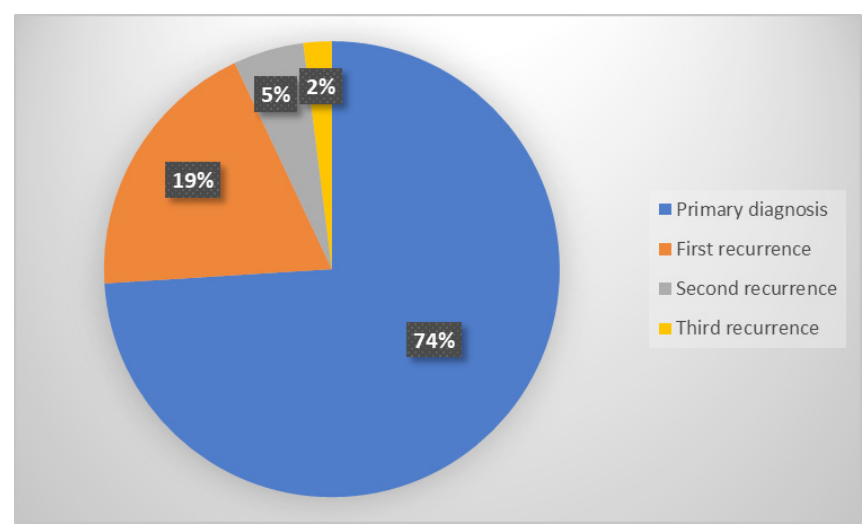

Fig. 1. The recurrence rate of PRTs

Following the statistical analysis, was found a partial correlation between tumour size and ultrasonographically estimated dimensions - 0.540 (95\% CI 0.295, 0.737, p <0.001), which is a high positive correlation. The appropriate use criteria (AUC) of ultrasonography was calculated to determine the retroperitoneal location of the tumours is 0.641 (95\% CI $0.541,0.740, \mathrm{p}<0.001$ ), the overall model quality being 0.54 . The edge characteristics of PRTs sonographic determined can suggest the benign or malignant type of the tumour $(\chi 2=9843, \mathrm{df}=1, \mathrm{p}<0.001)$. Thus, tumours located in the retroperitoneal space, with irregular edges have a higher probability of malignancy. A significant association was also identified between the consistency of the tumour (solid, cystic, mixed) and the benign or malignant type of the tumour ( $\chi 2=7526, \mathrm{df}=2, \mathrm{p}=0.023)$. Therefore, solid or mixed-textured tumours can be considered malignant. To determine the unicentric or multicentric type of the tumour, the ultrasonography demonstrated an integrative value of sensitivity and specificity of 0.644 (95\% CI 0.415 , 0.873, $\mathrm{p}<0.001)$.

In order to assess the treatment tactics, it is essential to determine the proximity of the tumour to the neighbouring organs. In the research group the most affected organs by retroperitoneal tumours were: the colon - in 15 cases (12.7\%), the small intestine - 22 cases (18.64), the main blood vessels - 23 cases $(19.49 \%)$, the pancreas - 17 cases $(14.40 \%)$, the kidney -9 cases $(7.62 \%)$, the adrenal gland -9 cases (7.62\%), the spleen -7 cases (5.93\%), the stomach -3 cases (2.54\%), the bladder -3 cases $2.54 \%)$. Ultrasonography of the abdominal cavity and retroperitoneal space used as a diagnostic test in determining the invasion of adjacent organs, demonstrated an integrative value of sensitivity and specificity as follows: involvement of the colon in the tumor process - AUC 0.767 (95\% CI 0.611, 0.922, p <0.001), the involvement of the small intestine in the process calculated AUC was 0.795 (95\% CI 0.672, 0.917, p <0.001), AUC calculation for determining the invasion of the pancreas by the tumour was 0.838 (95\% CI 0.705, 0.971, p <0.001), for kidney invasion -0.861 (95\% CI 0.699, 1.024, p <0.001), spleen - 0.567 (95\% CI 0.326, 0.808, p <0.001), main blood vessels (aorta, inferior vena cava, superior mesenteric artery and vein) -0.674 (95\% CI 0.532, 0.816, p <0.001) (tab. 1). 
Table 1. The informativeness of ultrasonography in PRTs diagnosis

\begin{tabular}{|c|c|c|c|}
\hline & & \multicolumn{2}{|c|}{ Ultrasonography } \\
\hline & & AUC & $\begin{array}{l}\text { Overall model } \\
\text { quality }\end{array}$ \\
\hline \multicolumn{2}{|c|}{$\begin{array}{c}\text { The localization of the } \\
\text { tumour }\end{array}$} & $\begin{array}{c}0.641 \\
(\text { IC 95\% 0.541, 0.740, } \\
p<0.001) .\end{array}$ & 0.54 \\
\hline \multicolumn{2}{|c|}{$\begin{array}{l}\text { The unicentric/multicen- } \\
\text { tric type of tumour }\end{array}$} & $\begin{array}{c}0.644 \\
(\text { IC } 95 \% 0.415,0.873, \\
p<0.001) .\end{array}$ & 0.42 \\
\hline \multicolumn{2}{|c|}{ The edge characteristics } & $\begin{array}{c}0.720 \\
(\text { IC } 95 \% 0.601,0.838 \\
p<0.001) .\end{array}$ & 0.49 \\
\hline \multirow{7}{*}{ 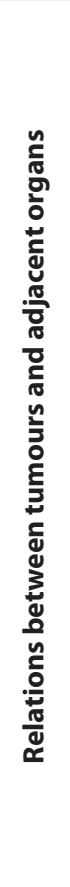 } & Colon & $\begin{array}{c}0.767 \\
\text { (IC 95\% 0.611, 0.922, } \\
p<0.001) .\end{array}$ & 0.61 \\
\hline & Small intestine & $\begin{array}{c}0.795 \\
(\text { IC } 95 \% 0.672,0.917, \\
p<0.001) .\end{array}$ & 0.67 \\
\hline & Pancreas & $\begin{array}{c}0.838 \\
(\text { IC 95\% 0.705, 0.971, } \\
p<0.001) .\end{array}$ & 0.71 \\
\hline & Kidney & $\begin{array}{c}0.861 \\
(\text { IC } 95 \% 0.699,1.024, \\
p<0.001) .\end{array}$ & 0.70 \\
\hline & Spleen & $\begin{array}{c}0.567 \\
(I C 95 \% 0.326,0.808, \\
p<0.001) .\end{array}$ & 0.33 \\
\hline & Adrenal gland & $\begin{array}{c}0.593 \\
(\text { IC 95\% 0.377, 0.808, } \\
p<0.001) .\end{array}$ & 0.38 \\
\hline & Blood vessels & $\begin{array}{c}0.674 \\
\text { (IC 95\% 0.532, } 0.816, \\
p<0.001) .\end{array}$ & 0.53 \\
\hline
\end{tabular}

All patients involved in this study underwent curative or diagnostic laparotomy. Although benign tumours were found, excision biopsy was performed in 12 patients $(35.3 \%$ $(95 \%$ CI $20.9,52.0))$ and excision of the tumour in 17 patients $(34.0 \%$ (95\% CI 22.1, 47.7)). Diagnostic laparotomy was performed in 15 patients (44.3\% (95\% CI 20.9, 52.0)) with histopathologically confirmed malignancies, and in 27 cases $(54.0 \%$ (95\% CI 40.3, 67.3)) the tumour was excised.

Although retroperitoneal cysts are in most cases benign, which was demonstrated in this study, all 6 cysts identified were benign, complete excision of the cysts was successful in only 2 cases $(4.0 \%(95 \%$ CI $0.8,12.2))$, in 4 cases that constituted $(11 \%(95 \%$ CI $20.9,52.0))$ the partial excision of the cysts was performed.

Non-Hodgkin's lymphomas with primary involvement of the retroperitoneal lymph nodes were confirmed in 7 cases: in 3 cases by excisional biopsy (8.8\% (95\% CI 4.1, 25.6)), and in 4 cases, when the disease was manifested by the presence of a tumour, solitary or lymph node conglomerate, the tumour was completely removed $(8.0 \%$ (95\% CI $2.8,17.9))$.

\section{Discussion}

PRTs are histologically heterogeneous benign and malignant neoplasms, being categorized on the basis of a single principle, the anatomical space where they develop - the retroperitoneal space [5].

Presented symptoms are often not specific and dependent on the anatomical site involved. The retroperitoneal sarcoma (RPS) usually grows as a mass, causing compression symptoms on other organs and a sense of abdominal discomfort, especially when it reaches a considerable volume. More frequently, RPS are incidental findings at the imaging tests performed for other reasons. Some of the most frequent symptoms are abdominal pain and discomfort, back pain, bowel obstruction, urinary and gynaecological symptoms. When the mass becomes bulky, it can be palpated externally [6-8].

A correct evaluation of the diagnostic images is paramount to stage the disease, establish the best therapeutic pathway and evaluate the surgical resectability.

Ultrasonography is an imaging diagnostic method that allows the evaluation of retroperitoneal tumours by providing information in an acceptable volume to assess subsequent diagnostic and treatment tactics. Due to the fact that the PRT becomes quite large at the time of diagnosis, the average tumour size of the patients in the study being 17.3 $\mathrm{cm}( \pm 10.5 \mathrm{~cm})$, the assessment of tumour size is difficult. In assessing the retroperitoneal or intraperitoneal localization as well as in determining the unicentric or multicenter tuple of the tumour, ultrasonography demonstrated integrative values of credible sensitivity and specificity, 0.641 and 0.644, respectively. Determining the margins and tumour texture by assessing tumour echogenicity at ultrasound examination plays a key role in determining the tactics and volume of surgical treatment applied. The surgical treatment applied can be palliative or radical. If curative surgery is expected, an assessment of the tumour's proximity to adjacent organs is essential. PRT being in $80 \%$ malignant cases, most often the tumour invades the adjacent organs. Ultrasonography, as a diagnostic method used in assessing organs invaded by the neoplastic process, has demonstrated integrative values of sensitivity and specificity of high veracity (the highest AUC being used to determine the invasion of the colon, small intestine, pancreas and kidney). An advantage of ultrasonography over other imaging methods used in the diagnosis of PRT is that it is available in all medical institutions, it is a harmless method that can be applied to all categories of patients and it is an inexpensive method that meets practically all the conditions for a screening investigation method.

\section{Conclusions}

Ultrasonography is an imaging diagnostic method that can be easily used in the diagnosis of PRT as a preoperative diagnostic step. It is an imaging method that provides information in acceptable volume for making a decision to approach a patient with a retroperitoneal tumour, but does not provide enough information to plan surgery. 


\section{References}

1. Sassa N. Retroperitoneal tumours: review of diagnosis and management. Int J Urol. 2020;27(12):1058-1070. doi: 10.1111/iju.14361.

2. Osman S, Lehnert BE, Elojeimy S, Cruite I, Mannelli L, Bhargava P, Moshiri M. A comprehensive review of the retroperitoneal anatomy, neoplasms, and pattern of disease spread. Curr Probl Diagn Radiol. 2013 Sep-Oct;42(5):191-208. doi: 10.1067/j.cpradiol.2013.02.001.

3. Wiseman JT, Ethun CG, Cloyd JM, Shelby R, Suarez-Kelly L, Tran T, Poultsides G, Mogal H, Clarke C, Tseng J, et al. Analysis of textbook outcomes among patients undergoing resection of retroperitoneal sarcoma: a multi-institutional analysis of the US Sarcoma Collaborative. J Surg Oncol. 2020;122(6):1189-1198. doi: 10.1002/jso.26136.

4. Huggett BD, Cates JMM. The Vanderbilt staging system for retroperitoneal sarcoma: a validation study of 6857 patients from the National Cancer Database. Mod Pathol. 2019;32(4)539-545. doi: 10.1038/s41379018-0166-8.
5. Bonvalot S, Rivoire M, Castaing M, Stoeckle E, Le Cesne A, Blay JY, Laplanche A. Primary retroperitoneal sarcomas: a multivariate analysis of surgical factors associated with local control. J Clin Oncol. 2009;27(1):317. doi: $10.1200 / J C O .2008 .18 .0802$.

6. Dumitra S, Gronchi A. The diagnosis and management of retroperitoneal sarcoma. Oncology (Williston Park). 2018 Sep 15;32(9):464-9.

7. Messiou C, Moskovic E, Vanel D, Morosi C, Benchimol R, Strauss D, Miah A, Douis H, van Houdt W, Bonvalot S. Primary retroperitoneal soft tissue sarcoma: imaging appearances, pitfalls and diagnostic algorithm. Eur J Surg Oncol. 2017 Jul;43(7):1191-1198. doi: 10.1016/j. ejso.2016.10.032.

8. Carbone F, Pizzolorusso A, Di Lorenzo G, Di Marzo M, Cannella L, Barretta ML, Delrio P, Tafuto S. Multidisciplinary management of retroperitoneal sarcoma: diagnosis, prognostic factors and treatment. Cancers (Basel). 2021 Aug 10;13(16):4016. doi: 10.3390/cancers13164016.

\section{Authors' ORCID iDs and academic degrees}

Victor Schiopu, MD, PhD Student, Assistant Professor - https://orcid.org/0000-0002-2244-0280

Vasile Turcanu, MD, PhD, Associate Professor - https://orcid.org/0000-0002-8999-8430

Nicolai Ghidirim, MD, PhD, Professor - https://orcid.org/0000-0003-1430-5203

\section{Authors' contributions}

VS interpreted the data and performed the analytical part of the work, drafted the first manuscript; VT and NG conceptualized the project, designed the research and revised the manuscript critically.

\section{Funding}

This study was supported by Nicolae Testemitanu State University of Medicine and Pharmacy. The trial was the authors' initiative. The authors are independent and take responsibility for the integrity of the data and accuracy of the data analysis.

\section{Ethics approval and consent to participate}

The research project was approved by the Research Ethics Committee of Nicolae Testemitanu State University of Medicine and Pharmacy (Protocol No 84, 20.06.2017).

\section{Conflict of Interests}

No competing interests were disclosed. 